\title{
NOTA SOBRE UMA NOVA TÉCNICA DE HEMOCULTURA PARA DIAGNÓSTICO PARASITOLÓGICO NA DOENÇA DE CHAGAS NA SUA FASE CRÔNICA*
}

\author{
Egler Chiari ** e João Carlos P. Dias **
}

Foram realizadas hemoculturas pela técnica de Mourão \& Mello em 16 pacientes na fase crônica da Doença de Chagas comprovando-se a infecçấo em 7 (43.7). A positividade por tubos de meio LIT semeados foi de $8,3 \%$.

Estes resultados confirmam os obtidos por Mourão \& Mello durante a padronização da ré̃erida técnica e recomendam o seu emprego como método diagnóstico.

A necessidade de padronizar novas técnicas para o diagnóstico parasitológico de pacientes na fase crônica da Doença de Chagas estimulou-nos a avaliar a sensibilidade da técnica preconizada por Mourāo \& Mello de hemoculturas no meio "LIT", utilizando-se um lavado de papas de hemácias.

\section{MATERIAL E MÉTODOS}

O trabalho foi realizado utilizando-se sangue colhicio em 16 pacientes com doença de Chagas crônica registrados no Posto "Dr. EMMANUEL DIAS" (Bambui, M.G.) do Instituto Oswaldo Cruz, Fiocruz, M. S. Todos possuíam nas suas respectivas ficha: clínicas resultados de reaçōes de Guerreiro e Machado reativas realizadas em épocas anteriores. Entre os 16 pacientes, 4 tiveram períado agudo da infecção comprovado pelo exame de sangue a fresco e 6 possuíam dados de comprovação parasitológica da infecção por xenodiagnóstico (Tabela 1 ).
Nesta observação seguimos basicamente a técnica preconizada por Mourão \& Mello (2).

Utilizamos tubos de ensaio do tipo "screw tube" de tamanho $18 \times 150$ com $5 \mathrm{ml}$ de meio LIT. A colheita do sangue foi feita em "Venoject tube" de $10 \mathrm{ml}$ (Modelo de $100 \times 15-16 \mathrm{~mm}$ ), número T-200, marca $\mathrm{J}$. Terumo Co. Ltd., adicionado de 30 unidades de heparina diluída em $0,5 \mathrm{ml}$ de solução salina fisiológica a $0,85 \%$. Cada $10 \mathrm{ml}$ de sangue heparinizado, após submetidos a técnica de Mourão \& Mello, foram semeados em 2 tubos com $5 \mathrm{ml}$ de meio LIT.

Após colher e semear o material de acordo com a técnica, as 'nemocuituras foram transportadas no mesmo dia para nosso laboratório em Belo Horizonte, incubadas a $28^{\circ} \mathrm{C}$ e examinadas após 45 e 60 dias.

Os resultacios apresentados nas tabelas 1 e 2 mostram que nos foi possivel comprovar 7 entre 16 pacientes $(43,7 \%)$, com uma positividade de $8,3 \%$ em relação ao número de tubos semeados.

* Prof. Adjunto do Departamento de Parasitologia ICB - UFMG e Pesquisador Centro Pesquisa René Rachou, INERu, FIOCRUZ - M.S.

* Professor Temporário do Departamento de Clínica Médica - (Disciplina de Doenças Infectuosas e Parasitárias) Fac. Medicina - UFMG.

*** Recursos do TC 46 - CNPq - Doenças de Cragas - MINIPLAN - FINEP.

Recebido para publicação em 18,4.75. 
TABELA I

Positividade de hemoculturas em 16 pacientes na fase crônica da Doença de Chagas realiz em meio LIT, segundo a técnica de MOURÃO \& MELLO

\begin{tabular}{|c|c|c|c|c|c|c|c|c|c|}
\hline \multirow{3}{*}{ 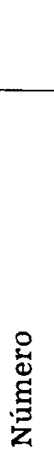 } & \multicolumn{6}{|c|}{ P A C I E N T E S } & \multicolumn{3}{|c|}{ NÚMERO DE TUBOS } \\
\hline & \multirow{2}{*}{ 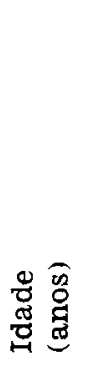 } & \multirow[b]{2}{*}{$\begin{array}{l}\stackrel{0}{x} \\
\underset{d}{0} \\
01\end{array}$} & \multirow{2}{*}{ 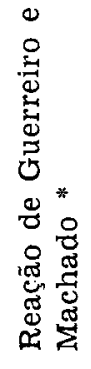 } & \multirow{2}{*}{ 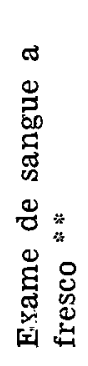 } & \multirow{2}{*}{ 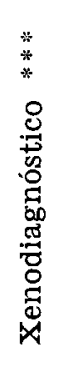 } & \multirow{2}{*}{ 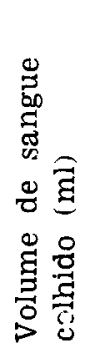 } & \multirow{2}{*}{ 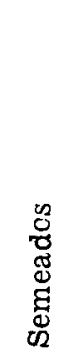 } & \multicolumn{2}{|c|}{$\begin{array}{c}\text { Positivos de } \\
\text { acorco com o } \\
\text { tempo de } \\
\text { incubação em } \\
\text { dias }\end{array}$} \\
\hline & & & & & & & & 45 & 60 \\
\hline 1 & 29 & $\bar{\varphi}^{\pi}$ & $\mathbf{R}$ & + & + & 30 & 6 & 1 & 0 \\
\hline 2. & 23 & $Q^{7}$ & $\mathbf{R}$ & $\ldots$ & .. & 40 & 8 & 1 & $\mathbf{0}$ \\
\hline 3 & 25 & $\varphi^{\prime}$ & $\mathbf{R}$ & + & - & 50 & 10 & $\mathbf{0}$ & $\mathbf{0}$ \\
\hline 4 & 47 & $\varphi^{\top}$ & $\mathbf{R}$ & $\ldots$ & $\ldots$ & 40 & 8 & 0 & $\mathbf{0}$ \\
\hline 5 & 26 & $Q^{7}$ & $\mathbf{R}$ & + & + & 30 & 6 & 0 & 0 \\
\hline 6 & 70 & 우 & $\mathbf{R}$ & .. & + & 45 & 9 & 1 & $\mathbf{0}$ \\
\hline 7 & 44 & 운 & $\mathbf{R}$ & $\ldots$ & + & 45 & 9 & $\mathbf{0}$ & 0 \\
\hline$\delta$ & 45 & $q$ & $\mathbf{R}$ & $\ldots$ & $\ldots$ & 45 & 9 & 1 & 0 \\
\hline 9 & 32 & q & $\mathbf{R}$ & $\ldots$ & $\ldots$ & 60 & 12 & 0 & 0 \\
\hline 10 & 33 & $q$ & $\mathbf{R}$ & $\ldots$ & $\ldots$ & 60 & 12 & 4 & 1 \\
\hline 11 & 50 & 우 & $\mathbf{R}$ & $\ldots$ & $\ldots$ & 45 & 9 & 0 & 0 \\
\hline 12 & 55 & $Q^{7}$ & $\mathrm{R}$ & $\ldots$ & $\ldots$ & 50 & 10 & 0 & 0 \\
\hline 13 & 36 & $q^{\top}$ & $\mathbf{R}$ & + & + & 40 & 8 & 0 & 1 \\
\hline 14 & 55 & $q^{7}$ & $\mathbf{R}$ & $\ldots$ & + & 50 & 10 & 0 & 2 \\
\hline 15 & 67 & $0^{\pi}$ & $\mathbf{R}$ & $\ldots$ & $\ldots$ & 45 & 9 & 0 & 0 \\
\hline 16 & 55 & $\sigma^{7}$ & $\mathbf{R}$ & $\ldots$ & $\ldots$ & 45 & 9 & 0 & 0 \\
\hline & $T$ & & & & & & 144 & 8 & 4 \\
\hline
\end{tabular}

* $\mathrm{R}=$ reagente

* realizado na fase aguda

*** Realizada na fase crônica 


\section{TABELA II}

Positividade de 'hemoculturas ,segundo técnicas de Mourão \& Mella, em pacientes na fase crônica da Doença de Chagas

\begin{tabular}{c|c|c}
$\begin{array}{c}\text { No de Pacientes } \\
\text { Examinados }\end{array}$ & $\begin{array}{c}\text { No de Pacientes com } \\
\text { Hemoculturas } \\
\text { positivas }\end{array}$ & $\%$ \\
16 & 7 & 43,7 \\
No tubos Semeados & No tubos Positivos & $\%$ \\
\hline
\end{tabular}

\section{COMENTÁRIOS E CONCLUSÕES}

Utilizando-se número equivalente de tubos com meio LIT para hemoculturas por paciente, Mourão \& Mello comprovaram $45,0 \%$ dos casos enquanto que obtivemos $43,7 \%$, percentagens bastante aproximadas. A positividade por tubo de hemocultura de Mourāo \& Mello foi de $17,0 \%$ obtendo-se 8,3\% no presente trabalho. Esta diferença pode em parte ser explicada porque fizemos as colheitas num só dia enquanto que
Mourão \& Mello (2) fazem as colheitas em diferentes dias o que parece aumentar as chances, à semelhança do que é feito na técnica de Schenone (3) para o xenodiagnóstico.

A análise dos resultados nos permtite concluir que a técnica de hemoculturas preconizada por Mourão \& Mello (2) foi reprodutível em nossas condições de trabalho, oferecendo boa perspectiva de emprego no diagnóstico parasitológico na fase crônica da Doença de Chagas.

\section{SUMMARY}

Performing hemocultures in "LIT" medium and Mourão \& Mello technique with blood of 16 pacients with chronic Chagas disease it was obtained positive results in 7 patients $(43,7 \%)$, representing 12 positives tubes $(8,3 \%)$. These findings support the view of Mourão \& Mello to indicate this diagnostic method. 


\section{REFERÊNCIAS BIBLIOGRAFICAS}

1. CHIARI, E. \& BRENER, Z. - Contribuição ao Diagnóstico parasitológico da Doença de Chagas na fase crônica. Rev. Ins Med. trop. S. Paulo 8: 134$-138,1966$.

2. MOURÃO, O. G. \& MELLO, O. G. Hemoculturas para o diagnóstico para- sitológico na fase crônica da Doença. de Chagas. (Em publicação).

3. SCHENONE, H. et al. - Valor del xenodiagnostico em la infeccion chagasica cronica. Bol. Chil. Parasit. 23: 149$-154,1968$. 